\title{
Éditorial : Incursions littéraires en anglais de spécialité
}

Literary inroads into English for Specific Purposes

\section{Anthony Saber}

\section{OpenEdition}

1 Journals

Édition électronique

URL : http://journals.openedition.org/asp/4868

DOI : 10.4000/asp.4868

ISSN : 2108-6354

Éditeur

Groupe d'étude et de recherche en anglais de spécialité

\section{Édition imprimée}

Date de publication : 1 novembre 2016

Pagination : 1-3

ISSN : 1246-8185

\section{Référence électronique}

Anthony Saber, «Éditorial : Incursions littéraires en anglais de spécialité », ASp [En ligne], 70 | 2016,

mis en ligne le 01 novembre 2016, consulté le 01 novembre 2020. URL : http://

journals.openedition.org/asp/4868; DOI : https://doi.org/10.4000/asp.4868

Ce document a été généré automatiquement le 1 novembre 2020.

Tous droits réservés 


\title{
Éditorial : Incursions littéraires en anglais de spécialité
}

\author{
Literary inroads into English for Specific Purposes
}

\author{
Anthony Saber
}

1 De prime abord, tout semble opposer les productions verbales issues des domaines spécialisés anglophones et la littérature : celle-ci procède d'une intention esthétique et désintéressée, celles-là reflètent des échanges entre acteurs utilisant la langue à des fins fonctionnelles (rendre une décision de justice, faire de la recherche scientifique, enseigner un domaine du savoir...) ; d'un côté, la noblesse perçue des " grands textes " de création, de l'autre, le caractère banal des textes utilitaires.

Pour autant, ces deux sphères ne sont pas totalement étanches l'une à l'autre. Il existe en effet, au sein même des textes spécialisés, et participant du mode de fonctionnement qui leur est propre, des formes que l'on pourrait qualifier de quasi littéraires, notamment des récits, qui sont dotés d'une finalité particulière en contexte spécialisé. Catherine Resche signale ainsi l'abondance de paraboles permettant de mettre en scène, sur un mode didactique, des postulats, des concepts ou mécanismes décrits par la science économique. Ce genre semble toujours vivace dans la période contemporaine, avec par exemple la parabole des "îles» d'Edmund Phelps (1969), ou encore celle du "surfeur de Malibu » de John Rawls (1988). Cette volonté démonstrative est également à l'œuvre lorsque l'économiste Frédéric Bastiat, pour critiquer les réflexes protectionnistes sur le mode de l'ironie, prend la plume en 1845 et rédige une « Pétition des fabricants de chandelles $»^{1}$, dans laquelle ceux-ci réclament, pour mieux garantir leur emprise sur le marché intérieur, que l'on ferme fenêtre et lucarnes, afin de bénéficier par là même d'une protection contre un redoutable « rival étranger » (celuici n'étant autre que le soleil). La "fiction économique didactique", déjà décrite précédemment par C. Resche (2004), et dont les Illustrations of Political Economy de Harriet Martineau, publiées entre 1832 et 1834, constituaient probablement les prodromes, illustre aussi cet intérêt constant des économistes pour la narration à dimension explicative. 
3 Le récit joue également un grand rôle dans le style qu'utilisent les scientifiques lorsqu'ils rapportent leurs travaux. Adoptant une perspective diachronique, David Banks établit une typologie des récits dans des articles scientifiques publiés dans les Philosophical Transactions au XVII ${ }^{\mathrm{e}}$ siècle et des articles parus dans les Proceedings of the Royal Society en 2015-2016. Des passages comportant des traits caractéristiques des récits sont observés dans les deux corpus, qu'il s'agisse de poser le paysage dans lequel s'inscrit l'étude, de rapporter des observations empiriques, de raconter les différentes étapes d'un protocole expérimental, ou encore d'effectuer des rappels bibliographiques. La permanence des schémas constatés, à quatre siècles de distance, illustre le fait que ces fonctions narratives semblent consubstantielles à tout compte rendu de travaux scientifiques.

4 La sphère financière n'échappe pas non plus à l'emprise des récits. Laurence Harris montre ainsi que la Banque d'Angleterre, par la bouche de son Gouverneur, ou par ses communiqués et ses rapports, déploie un récit performatif qui indique explicitement aux marchés financiers la trajectoire qu'ils devraient suivre. Cette technique d'annonces autoréalisatrices, nommée "forward guidance ", consiste à préformuler les anticipations des marchés, en décrivant à l'avance, de manière explicite, les événements qui les affecteront sur le plan de la politique monétaire. On peut opposer cette approche au mode de communication «delphique» qu'adopta jadis Alan Greenspan, ancien patron de la Federal Reserve américaine de 1987 à 2006, dont les conférences de presse et les dépositions devant le Congrès, souvent aussi peu explicites que les paroles de la Pythie grecque, pouvaient donner lieu à de multiples interprétations.

5 Thibault Marthouret décrit quant à lui l'apport de la poésie en cours d'anglais médical. La lecture de poèmes permet de développer les capacités d'interprétation, dont on sait toute l'importance, en médecine, dans le contexte de l'anamnèse. Écrire des poèmes amène aussi les étudiants à devenir plus créatifs, tout en améliorant la maitrise de certains schémas phonologiques et prosodiques. La "Médecine narrative " et les « Humanités médicales » constituent des champs de recherche et d'enseignement particulièrement prometteurs dans le domaine de l'anglais médical.

6 Ces articles attestent donc que des formes quasi littéraires sont bien présentes dans certains textes spécialisés, sur le mode de l'incursion: elles ne constituent certes pas l'ossature majeure des productions verbales spécialisées, mais y sont régulièrement activées pour remplir des fonctions spécifiques. On ne s'étonnera donc pas que l'étude des récits et de la mise en récit en contexte spécialisé anglophone fasse l'objet d'un intérêt croissant de la part des chercheurs en anglais de spécialité (voir par exemple Resche 2016), comme en témoigne le thème «Frontières narratives et poétiques en anglais de spécialité » du $37^{e}$ colloque du GERAS, organisé en 2016 par Gwen Le Cor à l'Université Paris 8 Vincennes-Saint-Denis. On peut ainsi se demander si, de même qu'une nation se constitue souvent autour d'un « roman national », certains domaines spécialisés ne possèdent pas un ou plusieurs "macro-récits" érigés en mythes fondateurs (devenir médecin, par exemple, n'est-ce pas entrer dans un récit ininterrompu de victoires contre la maladie, qu'Hippocrate commença il y a plus de vingt-cinq siècles ?).

7 Deux articles hors thème ainsi que plusieurs recensions, rédigées par John Humbley, Géraldine Gadbin-George, Marion Charret-Del Bove, Elizabeth Rowley-Jolivet, Dana Di Pardo Léon-Henri et Monique Mémet, complètent le présent numéro. Anne 
Brunon-Ernst s'intéresse à l'offre d'assurances proposée aux consommateurs lorsqu'ils achètent des billets d'avion sur Internet. Les compagnies aériennes parviennent à contourner partiellement la législation européenne en matière d'achat forcé, en ayant recours à des techniques de "conviction douce " (ou "nudges», concept décrit par Sunstein et Thaler en 2008). Catherine Resche réfléchit de son côté sur la notion d'engagement, particulièrement pertinente pour aborder de multiples facettes linguistiques, discursives et culturelles dans le domaine de l'économie.

La rédaction remercie chaleureusement les chercheurs qui ont accepté de relire les articles des numéros 69 et 70. Grâce à leurs commentaires, ils ont apporté une aide précieuse aux auteurs et à l'équipe éditoriale. Notre reconnaissance s'adresse à : Francisco Alfonso Almeida, Fabrice Antoine, Helen Basturkmen, Henri Béjoint, Jean-Claude Bertin, Ana Bocanegra-Valle, Pierre Busuttil $t$, Jean-Pierre Charpy, Marion Charret-Del Bove, Angela Chambers, Lynne Flowerdew, Peter Follette, Lise Fontaine, Marie-Hélène Fries, Geraldine Gadbin-George, Maurizio Gotti, John Humbley, Shaeda Isani, Ann M. Johns, Florence Lautel-Ribstein, Marie-Françoise Narcy-Combes, Jacqueline Percebois, Elsa Pic, Marie-Pierre Pouly, Isabelle Richard, Micaela Rossi, Elizabeth Rowley-Jolivet, Françoise Salager-Meyer, Thomas S. Miller, Catherine Resche, Gail Taillefer, Michel Van der Yeught, Christopher Williams, Séverine Wozniak.

Nos remerciements et nos pensées vont tout particulièrement vers Pierre Busuttil qui nous a brusquement quittés en août 2016.

\section{BIBLIOGRAPHIE}

MARTINEAU Harriet. 1832. Illustrations of Political Economy in 9 vols ( $3^{\text {rd }}$ ed). Londres : Charles Fox. Consulté le 3 octobre 2016 <http://oll.libertyfund.org/titles/1873>.

PHELPS, Edmund S. 1969. " The new microeconomics in inflation and employment theory ». American Economic Review: Papers and Proceedings 59, 147-160.

RAWLS, John. 1988. " The priority of right and ideas of the good ». Philosophy and Public Affairs 17/4, 251-76. Consulté le 3 octobre 2016 http://www.jstor.org/stable/2265400.

RESCHE, Catherine. 2004. " La théorie économique au détour de la fiction : le roman didactique ». In Petit, M. \& S. Isani, (dir.), Aspects de la fiction à substrat professionnel. Bordeaux : Université Victor Segalen Bordeaux 2, collection « Travaux 20.25 », 135-151.

RESCHE, Catherine (dir.). 2016. La mise en récit dans les discours spécialisés, Collection « Aspects linguistiques et culturels des discours spécialisés ». Berne : Peter Lang.

SUNSTEIN, Cass \& Richard THALER, R. 2008. Nudge: Improving Decisions About Health, Wealth and Happiness. New Haven : Yale University Press.

\section{NOTES}

1. Voir <http://bastiat.org/fr/petition.html> 


\section{AUTEUR}

\section{ANTHONY SABER}

Rédacteur en chef. anthony.saber@ens-cachan.fr 